\title{
Determinación de la viabilidad de semilla de Caesalpinia spinosa (Molina) Kuntze y su correlación con el contenido de goma y tanino
}

\author{
Determination of Caesalpinia spinosa (Molina) Kuntze seed viability and its \\ correlation with the content of rubber and tannin
}

\begin{abstract}
Rocio Guerrero Sosa ${ }^{1}$, Ignacio Lombardi Indacochea ${ }^{2}$, Héctor E. Gonzales Mora², Cecilia Figueroa Serrudo ${ }^{3}$ y Abelardo Calderón Rodríguez ${ }^{4}$
\end{abstract}

\begin{abstract}
Resumen
El propósito de esta investigación fue encontrar el método colorimétrico (test de tetrazolio o índigo carmín) que representa mejor la calidad de las semillas de tara (Caesalpinia spinosa) en menor tiempo, a diferencia de los ensayos de germinación tradicional. Además, se cuantificaron los contenidos de goma y taninos en cuatro estadios de maduración del fruto. La viabilidad de la semilla en cada estadio fue utilizada como un estimador del contenido de goma y taninos, mediante un análisis de correlación y regresión. Finalmente, al conocer el comportamiento del contenido de goma y tanino en los estadios de maduración evaluados se pudo determinar en qué estadio se obtiene el mayor contenido de ambos; resultado que podría ser de utilidad para los productores de ambas materias primas, con el fin de encontrar el momento apropiado de cosecha para un objetivo específico y lograr mayores rendimientos.
\end{abstract}

Palabras clave: tara; fruto; semilla; taninos; goma; germinación; viabilidad; test de tetrazolio; test del índigo carmín.

\begin{abstract}
The purpose of this research was to find the colorimetric method (tetrazolium and indigo carmine) wich determines the best quality of the seed in tara (Caesalpinia spinosa) in less time, unlike the traditional germination tests. Rubber and tannin content were quantified in four stages of fruit ripening and these contents were related to seed viability in each stage. Seed viability was used as an estimator of both, tannin and ruber content, using correlation and regression analysis. Finally, to know the content of rubber and tannin along fruit ripening is useful to find the appropriate time for fruit and seed harvest regarding specific purposes and achieve higher yields.
\end{abstract}

Key words: tara; fruits; seeds; tannin; rubber; germination; viability; tetrazolium test; indigo carmine test.

\footnotetext{
${ }^{1}$ Consultor independiente, Perú. E-mail: roguerrero001@gmail.com

${ }^{2}$ Facultad de Ciencias Forestales, Universidad Nacional Agraria La Molina, Perú.

${ }^{3}$ Facultad de Agronomía, Universidad Nacional Agraria La Molina, Perú.

${ }^{4}$ Facultad de Ciencias, Universidad Nacional Agraria La Molina, Perú.
} 


\section{Introducción}

La tara (Caesalpinia spinosa) es una planta originaria del Perú, utilizada desde la época prehispánica en la medicina folkclórica y en tiempos recientes como materia prima en el mercado mundial de hidrocoloides alimenticios y taninos. Se distribuye entre los $4^{\circ}$ y $32^{\circ}$ $S$ desde zonas áridas de Venezuela, Colombia, Ecuador, Perú, Bolivia llegando hasta el norte de Chile, creciendo con precipitaciones promedios anuales de 230 a $500 \mathrm{~mm}$, con biotemperaturas de 12 a $18^{\circ} \mathrm{C}$, pudiendo aceptar hasta $20^{\circ} \mathrm{C}$ (Flores et al. 2005).

Actualmente en nuestro país para conocer la calidad de las semillas forestales se viene realizando los ensayos de germinación tradicional, por lo que no se han encontrado publicaciones de tara donde haya sido probado otros métodos como la prueba de viabilidad con tetrazolio, método aceptado por la International Seed Testing Asssociation (ISTA) y usado mayormente en especies agrícolas. Otros métodos como la prueba de viabilidad con índigo carmín que son utilizados en otros países como España (Benito-Matías et al. 2004) y Argentina (Giorda et al. 1997) pero que aún no es aceptado por ISTA a pesar de ser un sistema bastante utilizado desde 1925 (Moreno-Álvarez et al. 2001). Por ello la importancia de investigar la aplicación de estos métodos colorimétricos en especies forestales, tomando en cuenta que estos métodos son más rápidos para conocer la calidad de la semilla, y así poder ir generando información en este campo sobre la tinción de semillas y/o embriones. Esto permitiría la predicción en los ensayos de germinación, encontrando así un método rápido, confiable, sencillo y reproducible.

De acuerdo a observaciones de semilla inmadura y madura se planteó que la producción de goma (endospermo) puede ir disminuyendo durante su maduración cuando llega a su volumen máximo. Ya que en muchas especies, el endospermo queda reducido a un remanente, al desempeñar una función nutricional del embrión, y en otras el endospermo no es consumido en el desarrollo del embrión, sino que queda para funcionar como tejido de almacenamiento (Hartmann 1995). Mientras que en el contenido de taninos lo que se planteó fue cómo es el aumento de este con respecto a su madurez, considerándose el color de la vaina como indicador de un mayor contenido según experiencias de los cultivadores. Investigación que contribuiría a saber en qué momento se logra tener un mayor contenido de goma y taninos durante los cuatro estadios de maduración evaluados y valorarlos cuantitativamente; para que así una empresa evalúe si es económicamente rentable aprovechar antes de la maduración total del fruto.

El propósito de ésta investigación fue: 1 . Identificar qué método colorimétrico (prueba de viabilidad del tetrazolio o índigo carmín) predice mejor la germinación. 2. Determinar el contenido de goma y taninos en cuatro estadios de maduración del fruto y 3 . Determinar si la viabilidad encontrada en cada estadio de maduración puede ser un indicador potencial del contenido de goma y taninos.

\section{Materiales y Métodos}

\section{Colección del material}

Los frutos de tara fueron recolectados en setiembre, octubre, noviembre y diciembre del año 2010, en la plantación de tara ubicada en Jayanca- Lambayeque, con coordenadas geográficas $06^{\circ} 23^{\prime} 27^{\prime \prime S} 79^{\circ} 49^{\prime} 19^{\prime \prime} \mathrm{W}$; perteneciente a la empresa La Puerta del Cielo SAC. La plantación tenía tres años de edad cuando se comenzaron las evaluaciones del año en mención, los árboles tenían una altura promedio de 2 metros. Previa a la recolección de los frutos, se realizó la marcación de los árboles a necesitar para obtener el número de semillas necesario para las evaluaciones.

Para la recolección del fruto se tomó en cuenta cuatro estadios de maduración, correspondientes a los meses de setiembre (estadio 1), octubre (estadio 2), noviembre (estadio 3) y diciembre (estadio 4), según la fenología de la especie en el lugar, desde que el fruto posee una longitud promedio semejante a la cosecha final 
y el mayor llenado del fruto por la formación de la semilla, es decir no se tomó en cuenta frutos planos. Los frutos de los tres primeros estadios se colectaron en planta y el fruto del cuarto estadio correspondiente a la cosecha final fue en suelo, alrededor del árbol. Se escogieron frutos enteros sin ningún tipo de ataque de insectos u hongos, o en estado de descomposición. Después de la recolección los frutos fueron trasladados al Laboratorio de Silvicultura, Laboratorio de Química Forestal (Facultad de Ciencias Forestales) y al Laboratorio de Fisiología vegetal (Facultad de Ciencias), de la Universidad Agraria La Molina - Lima (Perú) para los análisis respectivos.

\section{Análisis de laboratorio}

\section{Determinación de la estructura de la semilla}

La semilla madura se embebió en agua corriente por 48 horas con la realización de un pequeño corte en el extremo inferior de los cotiledones (opuesto al hilio), para facilitar la entrada de agua y lograr el ablandamiento de los tejidos. A la semilla hinchada y con la cubierta seminal ablandada se le retiró la cubierta seminal y el endospermo. A continuación, se retiró cuidadosamente los cotiledones, y se realizó un corte muy delgado con un bisturí al eje embrionario en dirección longitudinal para su observación al microscopio y se procedió a identificar la plúmula y radícula. Asimismo, se realizó un corte transversal a la cubierta seminal.

\section{Ensayo de germinación}

Se seleccionaron al azar 100 semillas y se dividieron en 4 repeticiones de 25 semillas cada una. Se utilizó como sustrato arena esterilizada humedeciéndola con agua destilada. Las semillas fueron sometidas a un pequeño corte mecánico en la parte inferior del cotiledón opuesto al hilio, para facilitar la absorción de agua en los estadios 2, 3 y 4 (excepto en el estadio 1 porque su cubierta aún no se endurecía), y se les aplicó fungicida en polvo (benlate). Las semillas fueron sembradas a una profundidad del doble de su espesor; se colocaron a temperatura de $24^{\circ} \mathrm{C}$ para conseguir la germinación. Las evaluaciones fueron realizadas cada dos días, durante 20 días. Se registraron las semillas que desarrollaron una raíz primaria para el cálculo de germinación (Lamarca et al. 2009), ya que la emisión de la radícula es la etapa final del proceso de germinación.

El porcentaje de germinación se calculó mediante la siguiente fórmula:

$\%$ de germinación $=\frac{N^{\circ} \text { de semillas germinadas }}{N^{\circ} \text { de semillas sembradas }} \times 100$

\section{Pruebas de viabilidad (tetrazolio e índigo carmín)}

Se utilizaron 4 repeticiones de 100 semillas tomadas al azar para cada prueba de viabilidad. Para la preparación de la solución de tetrazolio (TT), $\mathrm{C}_{19} \mathrm{H}_{15} \mathrm{~N}_{4} \mathrm{Cl}$, se empleó una solución acuosa al 0,05\% de 2,3,5-trifenil cloruro de tetrazolio, y para la preparación de la solución del índigo carmín (IC), $\mathrm{C}_{16} \mathrm{H}_{8} \mathrm{~N}_{2} \mathrm{O}_{8} \mathrm{~S}_{2} \mathrm{Na}_{2}$,se empleó una solución al 0,15\%, ambos con agua destilada.

Para la realización de ambas pruebas de viabilidad (TT e IC), las semillas fueron sometidas a un pequeño corte en uno de los extremos inferiores de la semilla (opuesto al hilio);luego, se sumergieron en agua corriente por un número determinado de horas según el estadio de madurez evaluado para ablandar el tegumento. Para el estadio 1, la semilla no fue remojada, debido a su gran contenido de humedad (al estar inmadura y en formación) y con un tegumento blando. Para el estadio 2, la semilla fue remojada por 29 horas, para el estadio 3, 36 horas y finalmente para el estadio 4, 48 horas. A continuación, el tegumento y el endospermo fueron removidos en cada semilla y los embriones fueron inmersos completamente en la solución de tetrazolio por 5 horas a $35^{\circ} \mathrm{C}$, en oscuridad. En la otra prueba con la solución de índigo carmín durante 18 horas a temperatura ambiente (alrededor de $20^{\circ} \mathrm{C}$ ). El endospermo removido, en forma de hojuelas, de las pruebas de viabilidad, fue destinado para la cuantificación de goma. Después de los tiempos mencionados, se decantó la solución y los embriones fueron lavados y cubiertos con agua destilada, sobre placas petri, para su inmediata evaluación. 
Para la evaluación de tinción del embrión, se procedió a desprender uno de los cotiledones cuidadosamente del nudo cotiledonar, y con la ayuda de un bisturí se realizó un corte longitudinal al eje embrionario que quedó inserto en uno de los cotiledones, para dejar expuesta la parte interna que comprende la plúmula, radícula y el hipocotilo llamado también "centro". Finalmente se evaluaron los cotiledones y el eje embrionario acondicionados. Para el test de TT se consideró que tejidos con coloración rojo brillante uniforme o rosa son típicos de tejido sano; tejidos con coloración blanca o amarilla son tejidos muertos; y tejidos con coloración rojo intenso son tejidos en deterioro (Fogaça et al. 2006). Para el test del IC los tejidos muertos se tiñen de azul, y los tejidos vivos permanecen incoloros (Benito-Matías et al. 2004).

Para la determinación del porcentaje de semillas viables para ambos test se tiene la siguiente relación:

$$
\text { Semillas viables }(\%)=\frac{N^{\circ} \text { embriones vivos }}{N^{\circ} \text { total de embriones }}
$$

\section{Cuantificación de goma}

La cuantificación de goma fue mediante dos métodos: (i) por solubilización de la goma en agua, denominado método gravimétrico (Guzmán 2009); y (ii) por la extracción del endospermo (goma) en forma de hojuelas, de las semillas acondicionadas a partir de las pruebas de viabilidad, denominándose método de hojuelas (método propuesto en la investigación).

\section{Cuantificación de tanino}

La vaina (fruto sin semilla) fue destinada para la cuantificación de taninos por el método cuantitativo del permanganato (adaptado del método de la A.O.A.C. Edición 14-1984). Donde 4,2 mg de taninos es igual a $1 \mathrm{ml}(0,1 \mathrm{~N})$ de permanganato de potasio.

\section{Diseño y análisis estadístico}

El diseño aplicado en el presente estudio fue completamente al azar (DCA); posteriormente los datos fueron evaluados estadísticamente mediante análisis de correlación y regresión lineal.

\section{Resultados \\ Características de los estadios de los frutos evaluados}

La longitud de los frutos evaluados del estadio 1 al 4 varía de $5-9 \mathrm{~cm}$ en promedio y tiene de 5 a 7 semillas. En los estadios 2 al 4 los frutos deben presentar un sonido característico de semillas sueltas en las vainas. Se describe otras características de los frutos recolectados, y su codificación de color (en la Figura 1) de acuerdo a la tabla Munsell para tejidos de plantas (Munsell 1977).

Estadio 1: La vaina es de color verde claro en ambas caras, cuya coordenada de color es de 7.5 GY (8/8/). En cuestión a su espesor es aproximadamente entre $0,7-1,0 \mathrm{~cm}$ y nunca frutos planos, demostrando así la mayor maduración de ésta con respecto a una vaina plana.

Estadio 2: La vaina es de color fucsia en la parte expuesta a la luz con algunas zonas de color mostaza, mientras que en la otra cara de la vaina correspondiente a la no expuesta debe ser de color mostaza y no de color verde, demostrando así su mayor maduración y disminución del contenido de humedad del fruto. Las coordenadas atribuidas para este estadio pueden ser dos: $2.5 \mathrm{R}(7 / 8 /)$ y $5 \mathrm{R}(5 / 8 /)$.

Estadio 3: La vaina es de color naranja en ambas caras en su mayoría, o sino solo en su parte expuesta y la no expuesta de color mostaza. Las coordenadas para éste estadio pueden ser dos: 5 YR (7/6/) y 5 YR (5/8/).

Estadio 4: La vaina es de color rojo intenso u oscuro, en un lado o en ambos; y este debe encontrarse en el suelo, o caer de la planta con un suave movimiento. Las coordenadas para este estadio pueden ser dos: $10 \mathrm{R}(4 / 10 /)$ y 5 YR $(4 / 8 /)$.

\section{Masa seca del fruto y de la semilla}

\section{Masa seca de la semilla}

Del estadio 1 al 2 se da una etapa de acumulación de reservas en el embrión y en el endospermo (Figura 2). Esta acumulación de reservas tiene su origen en los carbohidratos producidos por la fotosíntesis en las hojas y 


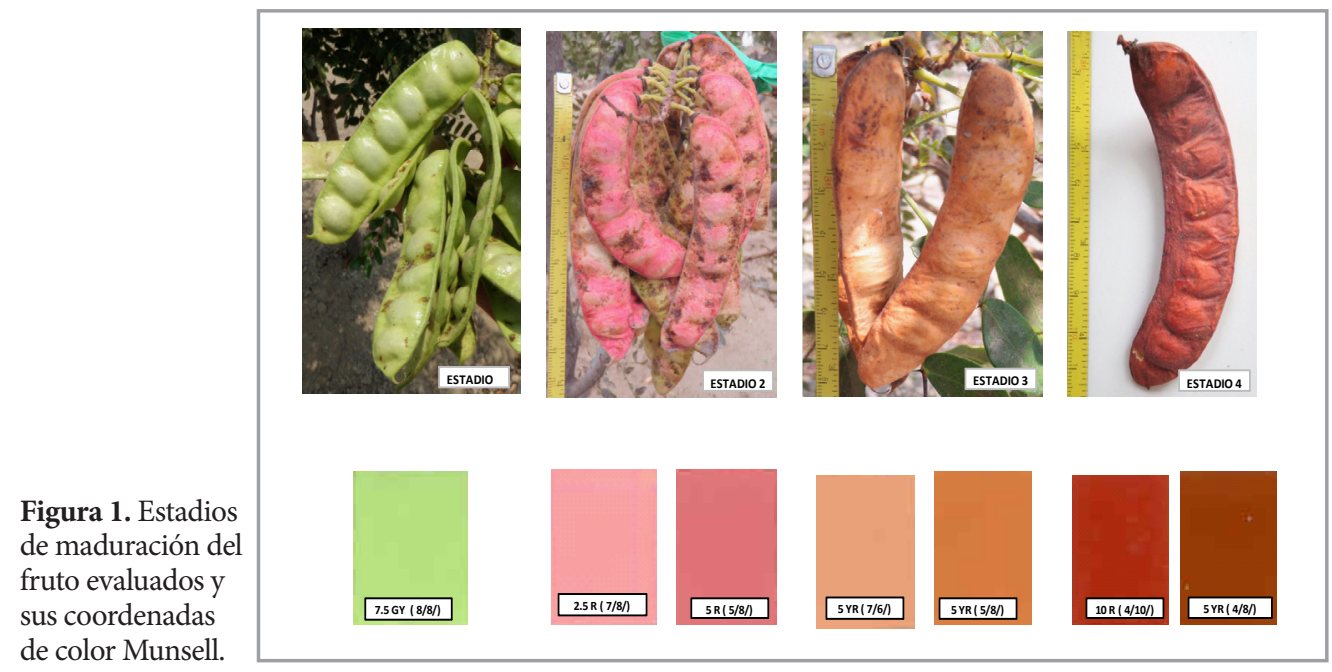

que son traslocados tanto al fruto como a la semilla, en donde se convierten en productos complejos de almacenamiento, en forma de carbohidratos, grasas y proteínas (Hartmann 1995). Al momento de evaluar embriones en el estadio 1 (pruebas de viabilidad), los cotiledones resultaron ser más delgados, que en los estadios 2, 3 y 4 , por lo que se demuestra que la acumulación de reservas es inminente en los cotiledones.

Luego del estadio 2 hay una estabilización de la masa seca de la semilla, como consecuencia de su independencia del fruto, por la ruptura del hilio que une la semilla y el fruto, demostrado por el sonido de semillas sueltas al agitar las vainas.

El endospermo (goma) también terminó su acumulación de reservas en el estadio 2 , como consecuencia de la independencia de la semilla del fruto, por lo que se considera que es a partir de este estadio en adelante, que el embrión consume parte del endospermo para continuar la acumulación de sus reservas según las disminuciones ligeras a partir del estadio 2 hasta llegar al estadio 4 (Figura 2).

Masa seca del fruto

La masa seca del fruto sigue una tendencia ascendente (Figura 2) por la acumulación de materiales de reserva; con respecto a la masa seca del fruto sin semilla (vaina) del estadio 1 al 2 se observa poca variación, mientras que a partir del estadio 2 al 4 hay un mayor aumento.

\section{Estructura de la semilla}

La semilla de tara es completa porque tiene las tres partes fundamentales (tegumento, endospermo y embrión) (Villanueva 2007). Su tegumento posee dos capas, una externa (testa) conformada por macroesclereidas, característica de las leguminosas; y una interna (tegmen). El embrión está conformado por el eje embrionario y los cotiledones, donde el eje embrionario a la vez está conformado por la radícula, hipocotilo y la plúmula (Figura 3). La plúmula en su origen son bipinnadas y al desarrollar forman los protófilos (hojas primarias) de la joven planta. La especie al ser dicotiledónea, posee dos cotiledones (excepcionalmente hasta tres) oblongos, aplanados, y en la superficie interna presenta nervaduras; ambos cotiledones encierran al eje embrionario, y la radícula sobresale en uno de los bordes externos (Flores 2010).

\section{Ensayos de germinación y viabilidad}

Los ensayos de germinación y viabilidad fueron realizados en los cuatro estadios de maduración del fruto como se muestra en la Figura 4. Donde la viabilidad fue hallada 


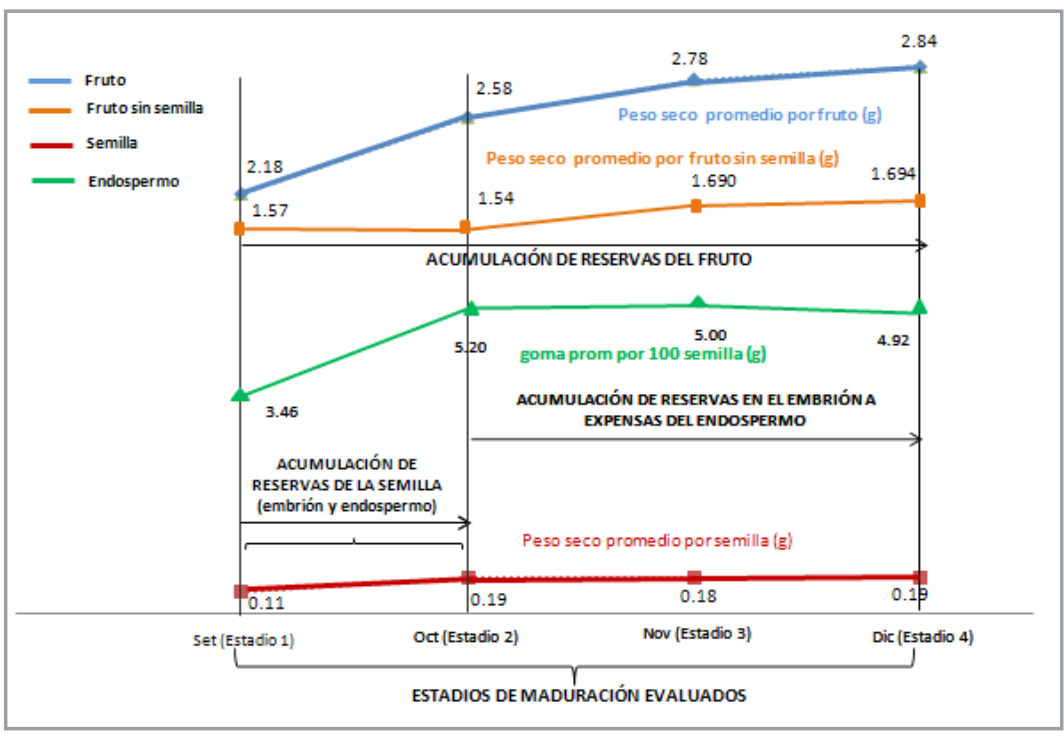

Figura 2. Comportamiento del peso seco del fruto, semilla y endospermo en cada estadio de maduración del fruto.

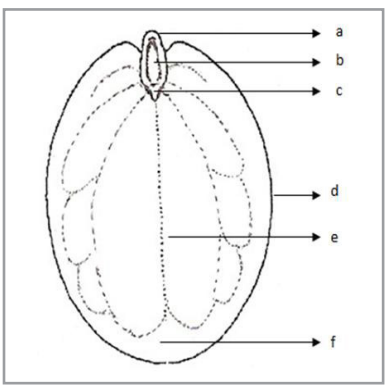

Figura 3. Estructura del embrión de la tara. Donde: (a-c) Eje hipocotilo radicular (Eje embrionario); (a) radícula; (b) hipocotilo (cilindro central o centro); (c) plúmula; (d) cotiledón; (e) nervaduras del cotiledón; (f) región distal del cotiledón.

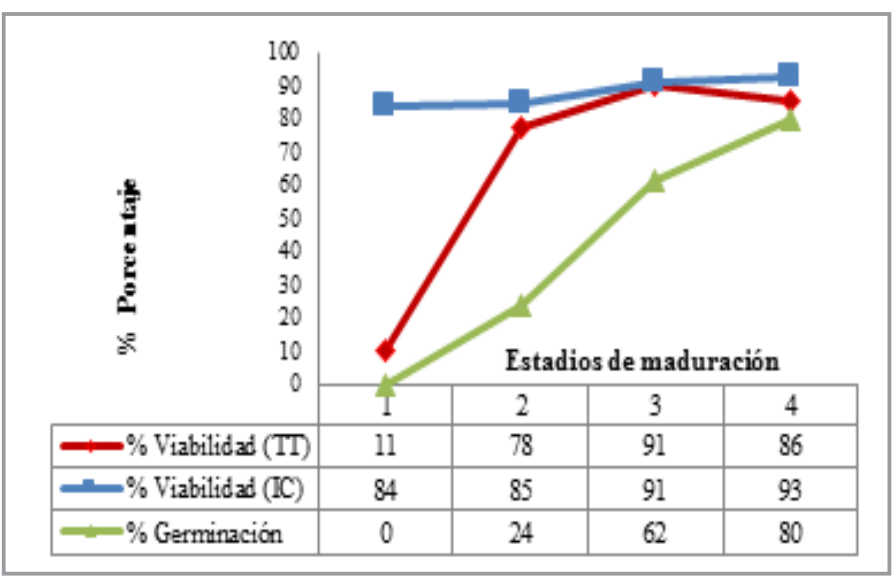

Figura 4. Porcentajes de germinación y viabilidad (tetrazolio e índigo carmín) para cada estadio de maduración del fruto. después de recolectado el fruto, es decir se obtuvo el potencial de semillas a germinar en cada mes evaluado, correspondiente a las características del fruto de un estadio determinado.

Por lo expuesto para realizar las correlaciones posteriores entre el ensayo de germinación y las pruebas de viabilidad, se consideró los valores del estadio 4 (Cuadro 1), de ambos ensayos por su proximidad con el ensayo de germinación.

\section{Clasificación de viabilidad}

Las semillas fueron clasificadas en seis patrones de tinción para cada test de viabilidad, los dos primeros corresponden asemillas viables, y los restantes a semillas no viables.

Descripción general de los patrones de tinción obtenidos en el test de tetrazolio:

Clase1: Viable.- Embriones con coloración rosa uniforme o poco variable, y todos los tejidos con aspecto normal y firme.

Clase 2: Viable.- Embriones con coloración 
rosa y menos del 50\% con coloración rojo intenso, sin proximidad al eje embrionario.

Clase 3: No Viable.- Embriones que presentan región cotiledonar con más del $50 \%$ con coloración rojo intenso o blanco lechoso muy próximo al eje embrionario, caracterizando tejido en deterioro o muerto respectivamente.

Clase 4: No Viable.- Embriones con coloración rosa y/o rojo intenso, con severas deformaciones y tejido flácido.

Clase 5: No Viable.- Eje embrionario o parte de éste con coloración blanco lechoso, y/o más del 50\% de la región cotiledonar con coloración blanco lechoso próximo al eje, indicando tejido muerto.

Clase 6: No Viable.- Eje embrionario o parte de éste con coloración rojo intenso, indicando grave proceso de deterioro. Embriones completamente con coloración rojo intenso o blanco lechoso, caracterizando tejido en deterioro o muerto respectivamente.

En la Figura 5 se presenta la tinción de embriones para las diferentes clases de viabilidad.

Descripción general de los patrones de tinción obtenidos en test del índigo carmín:

Clase 1: Viable.- Embriones completamente amarillos y todos los tejidos con aspecto normal y firme.

Clase 2: Viable.- Embriones con los bordes de los cotiledones de color azul y/o manchas de color azul $(<50 \%)$ de la región cotiledonar; $\mathrm{y}$ todos los tejidos con aspecto normal y firme.
Clase 3: No Viable.- Embriones con parte del eje embrionario de color azul, o embriones que presentan grandes zonas de color azul $(<50 \%)$, muy cerca próximo al eje embrionario, indicando tejido muerto.

Clase 4: No Viable.- Embriones con más del $50 \%$ de la región cotiledonar con coloración azul, y/o afectando el eje embrionario.

Clase 5: No Viable.- Embriones con severas deformaciones, pudiendo o no tener coloración azul.

Clase 6: No Viable.- Embriones con cotiledones completamente de color azul, típico de tejido muerto, pudiendo o no presentar el eje embrionario teñido.

En la Figura 6 se presentan la tinción de embriones para las diferentes clases de viabilidad.

\section{Contenido de goma}

Para conocer el comportamiento del contenido de goma (endospermo) en la semilla durante los cuatro estadios de maduración evaluados, se obtuvo el peso seco promedio de goma de 100 semillas, a partir del método de hojuelas para las pruebas de viabilidad (Cuadro 2). Se presenta la cuantificación de goma por obtenidos por el método de hojuelas y el método gravimétrico en el Cuadro 3.

El método elegido para la predicción del contenido de goma con las pruebas de viabilidad es el de hojuelas (Figura 7) ya que los valores de los últimos estadios de maduración analizados, se aproximan al resultado del análisis realizado el 30 de julio de 2010 en el Laboratorio de Pulpa y Papel para la cuantificación de goma,

\begin{tabular}{|c|c|c|c|}
\hline \multirow{2}{*}{ Repetición } & \multicolumn{3}{|c|}{ Ensayo } \\
\cline { 2 - 4 } & $\begin{array}{c}\text { \% Semillas } \\
\text { viables (TT) }\end{array}$ & $\begin{array}{c}\text { \% Semillas } \\
\text { viables (IC) }\end{array}$ & $\begin{array}{c}\text { \% Semillas ger- } \\
\text { minadas (Germi- } \\
\text { nación) }\end{array}$ \\
\hline 1 & 84 & 90 & 92 \\
\hline 2 & 86 & 91 & 76 \\
\hline 3 & 87 & 92 & 84 \\
\hline 4 & 87 & 97 & 68 \\
\hline Promedio & 86 & 93 & 80 \\
\hline
\end{tabular}

Cuadro 1. Resultados del ensayo de germinación y las pruebas de viabilidad para el estadio 4 .

\begin{tabular}{|c|c|}
\hline Estadio & $\begin{array}{c}\text { Peso seco promedio de } \\
\text { goma de } \mathbf{1 0 0} \text { semillas }(\mathrm{g})\end{array}$ \\
\hline 1 & 3,46 \\
\hline 2 & 5,20 \\
\hline 3 & 5,00 \\
\hline 4 & 4,92 \\
\hline
\end{tabular}

Cuadro 2. Contenidos de goma promedio en 100 semillas por el método de hojuelas. 


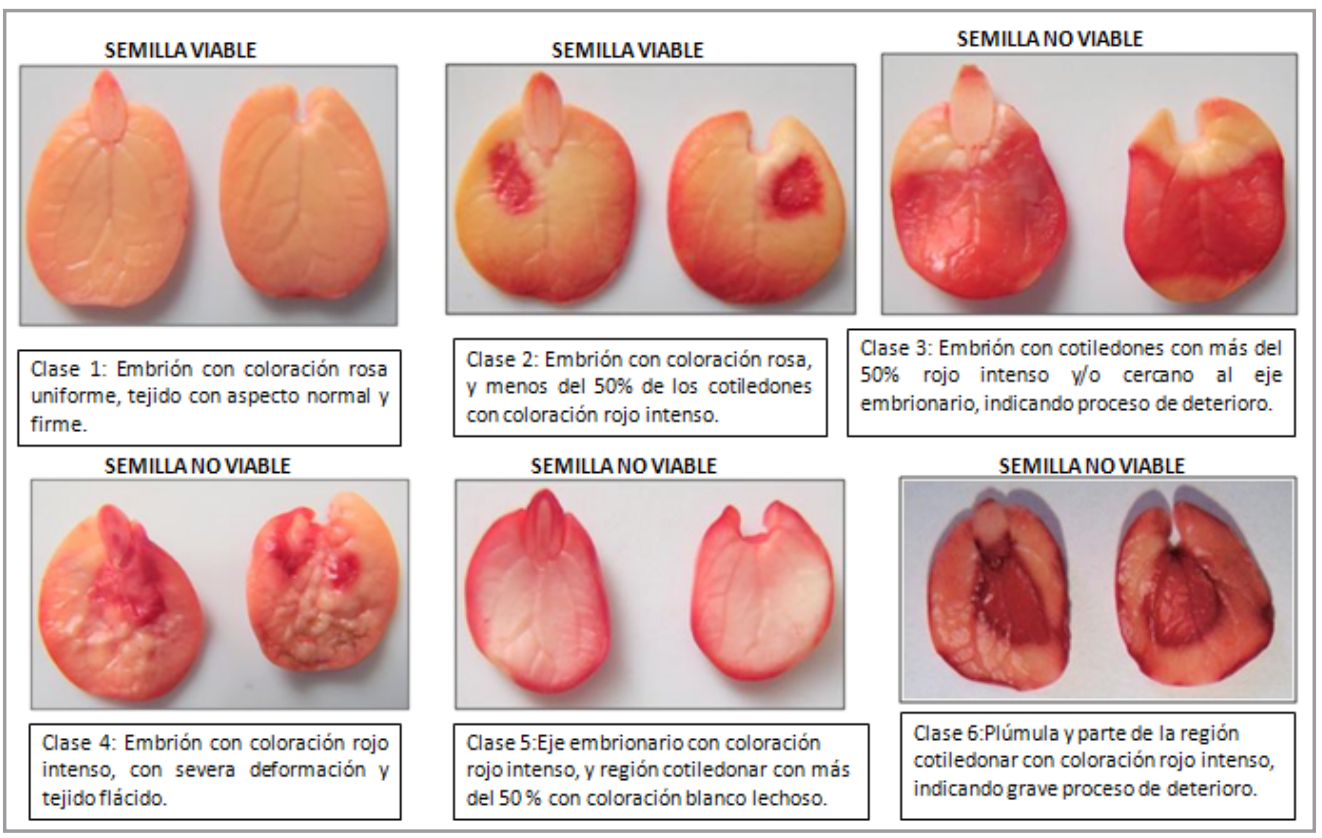

Figura 5. Patrones de tinción obtenidos de la prueba de viabilidad con el tetrazolio.

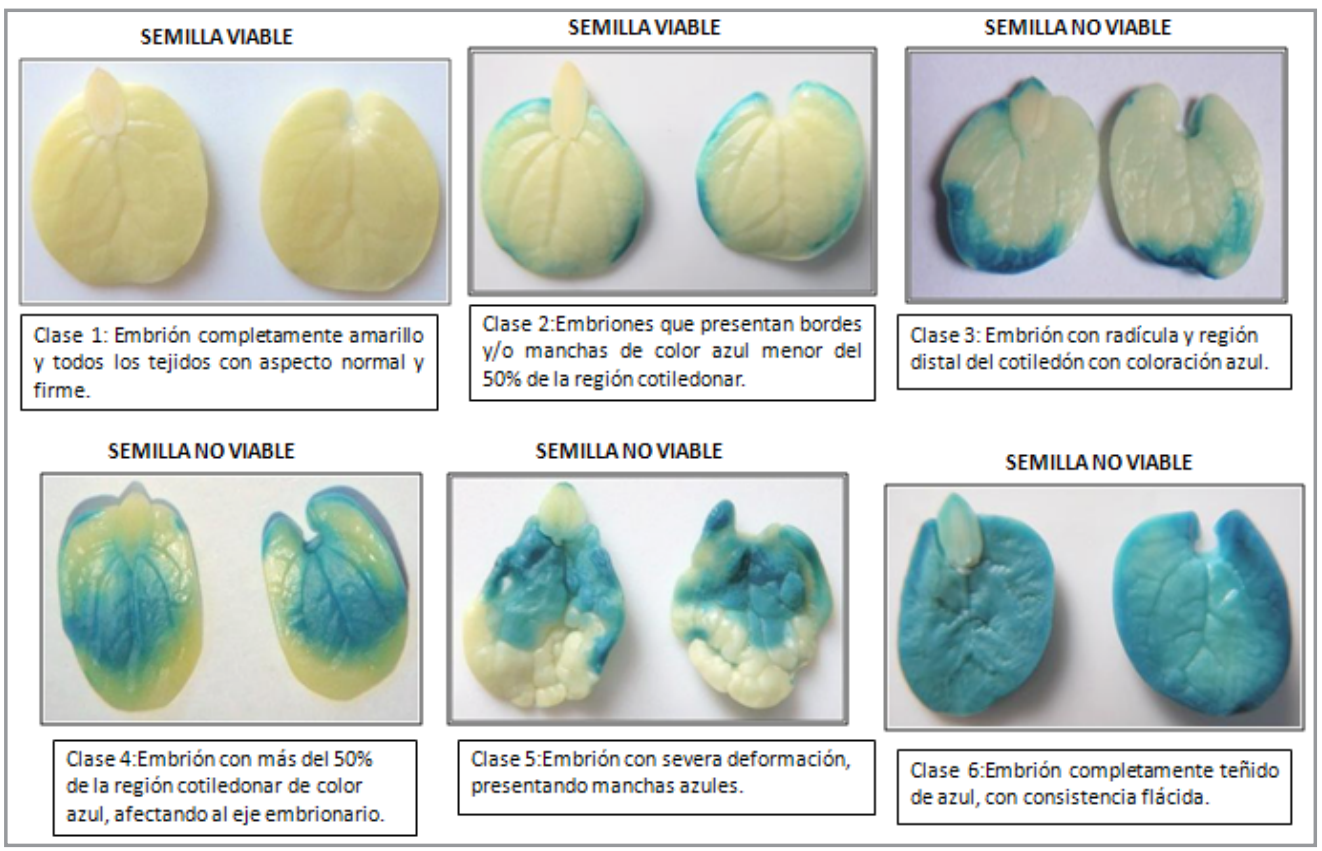

Figura 6. Patrones de tinción obtenidos de la prueba de viabilidad con el índigo carmín. 


\begin{tabular}{|c|c|c|}
\hline \multirow{2}{*}{ Estadio } & \multicolumn{2}{|c|}{$\%$ Goma } \\
\cline { 2 - 3 } & $\begin{array}{c}\text { Método de } \\
\text { Hojuelas }\end{array}$ & $\begin{array}{c}\text { Método Gravimé- } \\
\text { trico }\end{array}$ \\
\hline 1 & 32,96 & 38,98 \\
\hline 2 & 27,80 & 33,15 \\
\hline 3 & 27,81 & 30,15 \\
\hline 4 & 27,54 & 30,16 \\
\hline
\end{tabular}

Cuadro 3. Porcentaje de goma por el método de hojuelas y el método gravimétrico.

\begin{tabular}{|c|c|c|}
\hline Coeficiente & Tetrazolio (TT) & Índigo carmín (IC) \\
\hline $\mathrm{r}$ & 0.73 & 0.83 \\
\hline
\end{tabular}

Cuadro 4. Coeficiente de correlación del ensayo de germinación con las pruebas de viabilidad, ambos del estadio 4.

que dio un resultado de $28 \%$ por el método gravimétrico a partir de frutos recolectados en la cosecha efectuada en el mes de diciembre del año 2009.

\section{Contenido de tanino}

El fruto verde (estadio 1) posee 78,85\%, y los estadios 2, 3 y 4 poseen $80,07 \%, 80,29 \%$, $80,26 \%$ respectivamente, presentando estos últimos pequeñas diferencias en el contenido de taninos (Figura 8).

\section{Correlaciones}

\section{Germinación vs pruebas de viabilidad}

Ambos test presentan una correlación positiva fuerte con el ensayo de germinación, resultando ser métodos apropiados para conocer

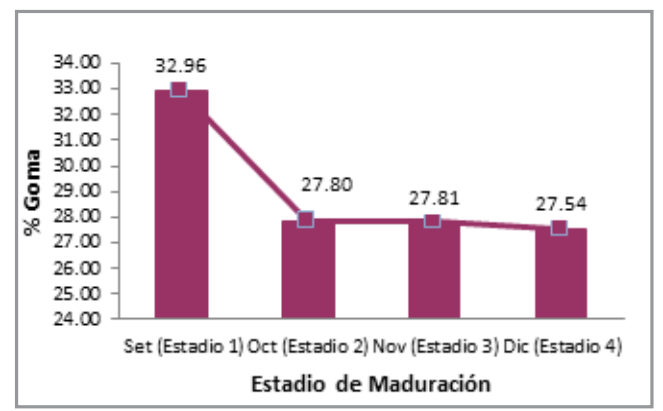

Figura 7. Porcentaje de goma para el método de extracción de hojuelas en los cuatro estadios de maduración evaluados. la calidad de la semilla en un menor tiempo (Cuadro 4).

Contenido de goma y taninos vs. pruebas de viabilidad

La relación de viabilidad versus los contenidos de goma y taninos se realizó desde el punto de vista productivo (Cuadro 5). Por esta razón, la viabilidad no se determinó después de la cosecha final (estadio 4), por ejemplo al primer, segundo o tercer mes después de cosechado el fruto; ya que el objetivo no era producir semilla de buena calidad para producir plantas, sino mostrar que tanto la maduración del embrión y de la semilla influían en el contenido de goma y tanino.

En el Cuadro 6 se muestran los resultados de los análisis de regresión lineal de las correlaciones con los valores presentados en el Cuadro 5. Se obtuvo una mayor correlación entre el test de tetrazolio con los contenidos de goma y taninos, además de validarse el modelo de estimación (ecuación) para la predicción de ambos contenidos.

\section{Cosecha apropiada según el estadio de mad- uración del fruto}

El enfoque de cosecha apropiada según el estadio de maduración del fruto, se le atribuye a los mayores rendimientos que se quieran obtener del contenido de goma y taninos, o la obtención del mayor peso del fruto; para lograr el máximo beneficio económico por el aprovechamiento de los frutos de un estadio determinado de maduración.

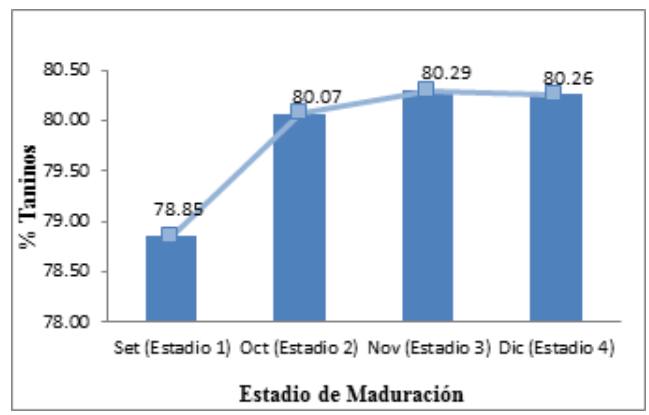

Figura 8. Porcentaje de tanino en los cuatro estadios de maduración evaluados. 


\begin{tabular}{|c|c|c|c|c|c|c|c|c|}
\hline \multirow[b]{2}{*}{ Relaciones } & \multicolumn{2}{|c|}{ Estadio 1} & \multicolumn{2}{|c|}{ Estadio 2} & \multicolumn{2}{|c|}{ Estadio 3} & \multicolumn{2}{|c|}{ Estadio 4} \\
\hline & $\begin{array}{c}\% \\
\text { Contenido }\end{array}$ & $\begin{array}{c}\% \text { Semilla } \\
\text { viable }\end{array}$ & $\begin{array}{c}\% \\
\text { Contenido }\end{array}$ & $\begin{array}{c}\text { \% Semilla } \\
\text { viable }\end{array}$ & $\begin{array}{c}\% \\
\text { Contenido }\end{array}$ & $\begin{array}{c}\% \text { Semilla } \\
\text { viable }\end{array}$ & $\begin{array}{c}\% \\
\text { Contenido }\end{array}$ & $\begin{array}{c}\% \text { Semilla } \\
\text { viable }\end{array}$ \\
\hline Goma vs TT & 32,39 & \multirow{2}{*}{11} & 27,89 & \multirow{2}{*}{78} & 28,01 & \multirow{2}{*}{91} & 27,60 & \multirow{2}{*}{86} \\
\hline Tanino vs TT & 78,85 & & 80,07 & & 80,29 & & 80,26 & \\
\hline Goma vs IC & 33,24 & \multirow{2}{*}{84} & 27,72 & \multirow{2}{*}{85} & 27,61 & \multirow{2}{*}{91} & 27,48 & \multirow{2}{*}{93} \\
\hline Tanino vs IC & 78,85 & & 80,07 & & 80,29 & & 80,26 & \\
\hline
\end{tabular}

Cuadro 5. Valores del contenido de goma, tanino y semillas viables.

\begin{tabular}{|c|c|c|c|c|c|c|}
\hline \multirow{2}{*}{ Ensayo } & \multirow{2}{*}{ Ecuación } & \multirow{2}{*}{$\mathbf{r}^{2}$} & \multirow{2}{*}{$\mathbf{r}$} & \multicolumn{2}{|c|}{ P-Value } & \multirow{2}{*}{$\begin{array}{l}\text { Modelo de } \\
\text { estimación }\end{array}$} \\
\hline & & & & Constante: $\boldsymbol{\beta}_{0}$ & Variable: $\beta_{1}$ & \\
\hline \multirow{2}{*}{ Tetrazolio } & $\%$ Goma en Hojuelas $=33.646-0.0695 \% \mathrm{TT}$ & 97.9 & 0.99 & 0.000 & 0.011 & Apropiado \\
\hline & $\%$ Taninos $=78.649+0.0183 \% \mathrm{TT}$ & 99.9 & 1.00 & 0.000 & 0.001 & Apropiado \\
\hline \multirow{2}{*}{$\begin{array}{l}\text { Índigo } \\
\text { carmín }\end{array}$} & $\%$ Goma en Hojuelas $=63.783-0.3938 \%$ IC & 44.1 & 0.66 & 0.148 & 0.336 & No apropiado \\
\hline & $\%$ Taninos $=69.815+0.1139 \%$ IC & 54.1 & 0.74 & 0.009 & 0.264 & No apropiado \\
\hline
\end{tabular}

Cuadro 6. Resultados de la regresión lineal del contenido de goma y tanino (en porcentaje) con las pruebas de viabilidad (\% semillas viables). Donde: $\mathrm{r}^{2}=$ coeficiente de determinación, $\mathrm{r}=$ coeficiente de correlación.

\section{Contenido de goma}

Se considera que el momento apropiado de recolección para la extracción de goma sería los frutos con las características de los estadios 2, 3 ó 4, ya que se emplea menos cantidad de semilla con respecto al estadio 1, para obtener las mismas cantidades de goma (Cuadro 7). Sin embargo con el estadio 2 se estaría aprovechando el fruto en menos tiempo a diferencia de los estadios 3 y 4 .

\section{Contenido de taninos}

Se considera que el momento apropiado de recolección para la extracción de tanino sería los frutos con las características de los estadios 3 o 4, ya que se emplea la misma cantidad de fruto para obtener $100 \mathrm{~g}$. de tanino, a diferencia de los estadios 1 ó 2, donde se emplea más fruto (Cuadro 8). Sin embargo con el estadio 3 se estaría aprovechando el fruto en menor tiempo.

\section{Contenido de goma y taninos}

Para aprovechar al máximo los contenidos de goma y taninos (tomando en cuenta los Cuadros 7 y 8), se tendría que recolectar frutos con las características 2 y 3 respectivamente. Con lo cual se estaría aprovechando el fruto en

\begin{tabular}{|c|c|c|}
\hline Estadio & $\begin{array}{c}\text { Peso de goma } \\
\text { seca (g) }\end{array}$ & $\mathbf{N}^{\circ}$ semillas \\
\hline Set (Estadio 1) & 100 & 923 \\
\hline Oct (Estadio 2) & 100 & 535 \\
\hline Nov (Estadio 3) & 100 & 551 \\
\hline Dic (Estadio 4) & 100 & 537 \\
\hline
\end{tabular}

Cuadro 7. Cantidad de semilla necesaria para obtener $100 \mathrm{~g}$ de goma seca.

\begin{tabular}{|c|c|c|}
\hline Estadio & $\begin{array}{c}\text { Peso de } \\
\text { tanino (g) }\end{array}$ & $\mathbf{N}^{\circ}$ frutos \\
\hline Set (Estadio 1) & 100 & 81 \\
\hline Oct (Estadio 2) & 100 & 81 \\
\hline Nov (Estadio 3) & 100 & 74 \\
\hline Dic (Estadio 4) & 100 & 74 \\
\hline
\end{tabular}

Cuadro 8. Cantidad de fruto necesario para obtener $100 \mathrm{~g}$ de tanino.

menos tiempo, pudiendo ser una alternativa económica interesante.

Peso seco del fruto

Si el objetivo es sólo vender fruto según su peso seco, las características del estadio 3 y/o 4, serían las apropiadas, ya que su peso aumenta considerablemente (Figura 2). 


\section{Discusión}

En el ensayo de germinación los porcentajes anteriores al estadio 4 y principalmente en los estadios 1 y 2 (etapa de acumulación de reservas por parte de la planta) fueron bajos, reafirmando lo mencionado por Hartmann (1995), que el proceso de desarrollo y acumulación de reservas debe efectuarse de manera apropiada y llegar a un grado mínimo de completamiento para la obtención de semillas de alta calidad.

Con respecto al estadio 4 , se obtuvo un porcentaje de germinación de $80 \%$, siendo este más próximo al de las pruebas de viabilidad (TT con $86 \%$, IC con 93\%) (Figura 4), resultando ser este estadio donde la semilla alcanza el máximo poder germinativo. El cual fue denominado como punto de maduración fisiológica, lo que posibilita que las semillas cosechadas sean las de máxima calidad (Bergemann de Aguiar et al. 1993).

De los valores obtenidos en el Cuadro 2 se identificó que del estadio 1 al 2, el contenido de goma en la semilla aumenta, es decir la acumulación de goma continuó hasta el estadio 2, considerándose este último como el punto máximo del contenido de goma. Mientras que para los estadios 3 y 4 los valores decrecen, posiblemente porque el embrión continúa su acumulación de reservas a expensas del tejido nutritivo del endospermo, ya que la semilla no tiene la disposición de alimento de la planta a consecuencia de su independencia a partir del estadio 2 , ocasionando pequeñas disminuciones.

Se observa un mayor rendimiento del contenido de goma en el estadio 1 , sin embargo este valor no significa que hay mayor contenido de goma, sino por el contrario hay un menor contenido, como se vio anteriormente en la Figura 2. Este mayor rendimiento se da porque los métodos de cuantificación están basados en la determinación de goma seca sobre el peso seco de la semilla, y no considerando el número de semillas que contiene este peso seco. Por lo tanto, si ponemos como ejemplo un kilogramo de semilla seca en estadio 1 , el número de semillas será mayor, ya que la semilla por unidad pesa menos $(0,11 \mathrm{~g})$ que en los estadio 2,3 o $4(0,19$ g; 0,18 g y $0,19 \mathrm{~g}$ respectivamente); siendo este mayor número de semillas en el estadio 1 , lo que conlleva a obtener un mayor rendimiento de goma. Es por ello que la interpretación de estos contenidos de goma (en base seca), expresados en porcentaje, debe ser cuidadosa de acuerdo al objetivo; ya que son como normalmente se expresan.

Se encontró que en frutos verdes inmaduros (estadio 1), hay cantidades considerables de taninos (compuesto fenólico) como lo menciona Valencia (1995) y Esau (1987) y además que estas concentraciones son mayores con respecto a la maduración (Llaudy et al. 2005) y no desaparecen durante el proceso de maduración; sino por el contrario demuestra ser parte de la acumulación de reservas en el fruto y su estabilización con el peso seco del mismo.

Además se descarta que el color constituya ser un indicador directo del contenido tánico, es decir que según los cultivadores haya que esperar la cosecha final para obtener más tanino.

\section{Conclusiones}

Ambas pruebas de viabilidad (tetrazolio $\mathrm{r}=0,73$ e índigo carmín, $\mathrm{r}=0,83$ ) comparados con los ensayos de germinación son buenos estimadores del potencial germinativo.

El contenido de goma aumenta del estadio 1 al 2, mientras que del estadio 2 al 4 hay una ligera disminución.

El contenido de taninos aumenta con la maduración del fruto. Principalmente entre los estadios 1 y $2(78,85 \%$ a $80,07 \%$ respectivamente), con respecto a los estadios 3 y 4 (80,29\% y $80,26 \%$ respectivamente). Este aumento tiene relación directa con el aumento del peso del fruto.

El contenido de goma y taninos puede ser estimado según las pruebas colorimétricas, siendo el test de tetrazolio el mejor estimador que podría constituirse como modelo apropiado para predecir ambos contenidos: contenido de goma $(r=0.99, p<0.05)$ y contenido de tanino $(\mathrm{r}=1.00, \mathrm{p}<0.05)$. 
Para la extracción de goma el momento más apropiado de recolección son los frutos con las características del estadio 2. Para la extracción de taninos es el estadio 3. Ambos con la finalidad de aprovechar el fruto en menor tiempo.

Para la venta por peso seco del fruto son apropiadas las características del estadio 3 y/o 4 , ya que el peso seco aumenta considerablemente.

\section{Agradecimientos}

A la empresa La Puerta del Cielo SAC, por el financiamiento y apoyo de campo en su plantación para la realización de la presente investigación.

\section{Bibliografía}

Benito-Matías, L; Herrero, I; Peñuelas, J. 2004. Aplicación de métodos colorimétricos para la determinación de la viabilidad en semillas de Pinus pinea: test de tetrazolio e índigo carmín. Sociedad Española de Ciencias Forestales n.17. España. p: 23-28.

Bergemann de Aguiar, I; Piña-Rodrigues, F; Balistiero-Figliolia, M. 1993. Sementes Florestais Tropicais. Associação brasilera de tecnologia de sementes. Brasília-DF. p: 215-217.

Esau, K. 1987. Anatomía de las plantas con semilla. Primera edición. Argentina. p. 33.

Flores, F; Chávarry, L; Vega, D. 2005. Criterios y pautas para la selección de árboles plus. Cajamarca - Perú. Pág. 21-22.

Flores, M. 2010. Determinación de la estructura de la semilla de tara Caesalpinia spinosa. Informes en el Laboratorio de Biología. Facultad de Ciencias. UNALM. Lima-Perú.

Fogaça, C; Malavasi, M; Zucareli, C; Malavasi, U. 2006.Aplicação do teste de tetrazólio em sementes de Gleditschia amorphoides Taub. Caesalpinaceae. Rev. Bras. Sementes. vol.28, n.3, pp. 101-107. Disponible en: http://www.scielo.br/scielo.php?script=sci_arttext\&pid=S0101-31222006000300015\&lng=es\&nrm=iso

Giorda, LM; Baigorri, HEJ. 1997. El cultivo de soja en Argentina. Cap.4. Calidad de la semilla. Agro 4 de Córdoba. Córdoba. Disponible en: http://www.planetasoja.com.ar/index.php?sel- menu $=0 \&$ sec $=2 \&$ tra $=2211 \&$ tit $=2229$

Guzmán, D. 2009. Cuantificación de goma de tara Caesalpinia spinosa por el método gravimétrico. Informes en el Laboratorio de Pulpa y Papel. Facultad de Ciencias Forestales. UNALM. Lima-Perú.

Hartmann, H. 1995. Propagación de plantas. Principios y prácticas. Segunda edición. México. p: 81-84.

Lamarca, E; Mota-Leduc, S; Barbedo, C. 2009. Viabilidade e vigor de sementes de Caesalpinia echinata Lam. (pau-brasil- Leguminosae) pelo teste de tetrazólio. Rev. Brasil. Bot. 32(4). p. 794.

Llaudy, M; Canals, R; Cabanillas, P; Canals, J; Zamora, F. 2005. La maceración prefermentativa en frío. Efectos en la extracción del color y los compuestos fenólicos, e influencia del nivel de maduración de la uva. Departamento de Bioquímica y Biotecnología, Unidad de Enología. Revista de Enología. N 60. Catalunya, España. Disponible en: http://www.acenologia.com/ciencia72_2.htm

Moreno-Álvarez, T; Benito-Matías, L; Herrero-Sierra, N; Domínguez-Lerena, S; Peñuelas-Rubira, J. 2001. Estudio de nuevos métodos de determinación de la viabilidad de las semillas forestales: test de electroconductividad e índigo carmín. Comparación con el test de tetrazolio y su aplicación a Pinus pinaster y Pinus halepensis. Centro de Mejora Forestal "El serranillo". Ministerio de medio ambiente. Guadalajara. España. p: 653-658.

Munsell, AH. 1977. Munsell color charts for plant tissues. Segunda edición.

NORMA A.0.A.C. 1984. Método cuantitativo del Permanganato. Adaptación del método para la cuantificación de taninos en la vaina de tara.

Valencia, C. 1995. Fundamentos de fitoquímica. Primera edición. México.

Villanueva, M. 2007. La tara "El oro verde de los Incas". Universidad Agraria La Molina. Lima-Perú. 